\title{
A pipeline for the de novo assembly of the Themira biloba (Sepsidae: Diptera) transcriptome using a multiple k-mer length approach
}

\author{
Dacotah Melicher ${ }^{1 *}$, Alex S Torson ${ }^{1}$, lan Dworkin² and Julia H Bowsher ${ }^{1}$
}

\begin{abstract}
Background: The Sepsidae family of flies is a model for investigating how sexual selection shapes courtship and sexual dimorphism in a comparative framework. However, like many non-model systems, there are few molecular resources available. Large-scale sequencing and assembly have not been performed in any sepsid, and the lack of a closely related genome makes investigation of gene expression challenging. Our goal was to develop an automated pipeline for de novo transcriptome assembly, and to use that pipeline to assemble and analyze the transcriptome of the sepsid Themira biloba.

Results: Our bioinformatics pipeline uses cloud computing services to assemble and analyze the transcriptome with off-site data management, processing, and backup. It uses a multiple k-mer length approach combined with a second meta-assembly to extend transcripts and recover more bases of transcript sequences than standard single k-mer assembly. We used 454 sequencing to generate 1.48 million reads from cDNA generated from embryo, larva, and pupae of T. biloba and assembled a transcriptome consisting of 24,495 contigs. Annotation identified 16,705 transcripts, including those involved in embryogenesis and limb patterning. We assembled transcriptomes from an additional three non-model organisms to demonstrate that our pipeline assembled a higher-quality transcriptome than single k-mer approaches across multiple species.

Conclusions: The pipeline we have developed for assembly and analysis increases contig length, recovers unique transcripts, and assembles more base pairs than other methods through the use of a meta-assembly. The T. biloba transcriptome is a critical resource for performing large-scale RNA-Seq investigations of gene expression patterns, and is the first transcriptome sequenced in this Dipteran family.
\end{abstract}

Keywords: Multiple k-mer, de novo assembly, Sepsidae, Transcriptome, Pipeline, Cloud computing

\section{Background}

The Sepsidae family of flies consists of over 200 species with a global distribution [1]. Sepsids are a model system for the investigation of sexual selection and how it affects courtship and sexual dimorphism [2]. Sepsids have complex courtship behaviors that include elements of male display, female choice, and sexual conflict [3-6]. Specialized male traits have evolved alongside these complex courtship behaviors. Sexual selection has resulted in the evolution of modified forelimbs, body size, and abdominal appendage-like structures, which are articulated and have

\footnotetext{
* Correspondence: Dacotah.Melicher@ndsu.edu

'Department of Biological Sciences, North Dakota State University, 1340

Bolley Drive, 218 Stevens Hall, Fargo, ND 58102, USA

Full list of author information is available at the end of the article
}

long bristles attached to their distal ends [7-15]. Nextgeneration sequencing in combination with gene expression analysis has the potential to answer multiple questions including: how new morphologies evolve, whether shared developmental mechanisms underlie traits that have evolved multiple times, what the genetic basis of sexual dimorphism is and how to resolve the phylogenetic relationships within Sepsidae. Despite the potential of sepsids as a model to test a wide variety of evolutionary hypotheses, almost no molecular resources exist in this family, nor are any genomes or EST databases available.

Most Dipteran families have few genomic resources compared to drosophilids and mosquitoes. Sepsids shared a common ancestor with Drosophila melanogaster and houseflies between 74 and 98 MYA, and are not closely 
related to any taxon with significant genomic resources $[16,17]$. A detailed investigation of the even-skipped locus revealed that approximately twice as many nucleotide substitutions exist between coding regions of $D$. melanogaster and sepsid species as exists between $D$. melanogaster and the most distantly related Drosophila species [18]. The Sepsidae are a sister taxon to the Tephritoidea or true "fruit flies," which contains four species with genomic and transcriptomic resources [19-22], but these are not as well annotated as Drosophila and the level of sequence similarity with sepsids is unknown. A sepsid transcriptome would not only facilitate gene expression studies across the Sepsidae, but would also enhance comparative bioinformatics within Diptera.

For non-model organisms, the challenge of gene discovery no longer resides in a dearth of sequence data, but from the computational challenges of large and complex datasets [23]. This challenge is particularly true for de novo assembly, which is more computationally intensive than syntenic assembly via mapping to a reference genome. Another hurdle to de novo assembly is recovering rare transcripts from a datasets with heterogeneous sequence coverage. Assemblies that combine multiple k-mer lengths generally recover a greater number of unique transcripts during de novo assembly than single k-mer approaches [24,25], but with additional potential for mis-assembly. Although both cloud computing and multiple k-mer approaches are widely available, they have not been employed as broadly as referencebased pipelines because some programing knowledge is required.

Our objectives were two-fold: 1) to construct a general purpose de novo transcriptome assembly pipeline that compares the output of multiple programs and automatically analyzes this data for downstream applications, and 2) to use that pipeline to assemble the transcriptome of the sepsid T. biloba. Our pipeline uses VelvetOases and Trinity for the initial assembly and constructs a meta-assembly with CAP3 followed by analysis with various downstream programs, including BLAST and Blast2GO [26-29]. The pipeline functions on a low-cost cloud computing network, and can be operated from a standard desktop computer. In addition to assembling the de novo transcriptome of the sepsid fly T. biloba, we used this pipeline to re-assemble previously published transcriptomes that used both 454 and Illumina sequencing platforms. Compared to the standard single k-mer assembly, our pipeline assembles longer contigs and more base pairs in all four species. By comparing annotated transcripts from different assemblies of the $T$. biloba transcriptome, we demonstrate that our pipeline recovers a greater number of transcripts than standard approaches by pooling unique transcripts from multiple assemblies.

\section{Results}

\section{General overview of computational pipeline}

This pipeline was designed to automate a large number of intermediate bioinformatic activities such as trimming and filtering reads, converting sequence files through various formats, performing a large number of sequential assemblies using different assemblers and parameters, and formatting the output for downstream use (Figure 1). This pipeline was also designed to circumvent what have traditionally been significant limitations for small research groups, a lack of computing facilities and programing knowledge. This pipeline, while functional on a local network, is designed to make use of virtual cloud computing units, which provide scalable resources with direct interaction. Our pipeline produces intermediate products that are compatible with graphical user interface (GUI) based platforms such as The iPlant Collaborative and Galaxy, so that researchers can use these interfaces for downstream applications if desired [30-33].

We used this pipeline to perform the de novo assembly of the $T$. biloba transcriptome, the first transcriptome assembly for any species for the family Sepsidae. We also used the pipeline to re-assemble archived RNA-seq reads from other studies to assess the performance of the multiple k-mer length assembly process compared to a single k-mer assembly. Archived sequence from an arthropod (the milkweed bug, Oncopeltus fasciatus: [SRR:057573]), a plant (Silene vulgaris: [SRR:245489]), and a mammal (the ground squirrel Ictidomys tridecemlineatus: [SRR:352220]) were selected to test the performance of the pipeline across taxa and genome sizes. Each of these data sets consists of 454 sequence reads of approximately 3.2-4x coverage, the same coverage as our $T$. biloba data set. The $O$. fasciatus and $S$. vulgaris sequence reads were generated for de novo assembly of the entire transcriptome of the organism while the I. tridecemlineatus sequences were generated for differential expression analysis [34-36].

\section{Cloud computing network and data management}

All of the data presented here were generated using Amazon Web Services Elastic Cloud Compute (AWS EC2) using a Debian Linux operating system (version 6.0.3). Software, sequence reads, reference assemblies, and other files are stored persistently on AWS Elastic Block Storage (EBS) volumes for the purpose of off-site backup, reduced network traffic, and storage. Data produced by the pipeline may be parsed and manipulated further through AWS or downloaded locally as needed. As presented here, the pipeline runs software in series. However, it is simple to create many duplicate systems through AWS, which may then run the processes in parallel.

Cloud computing instances were initialized using memory-optimized architecture to memory requirements the 


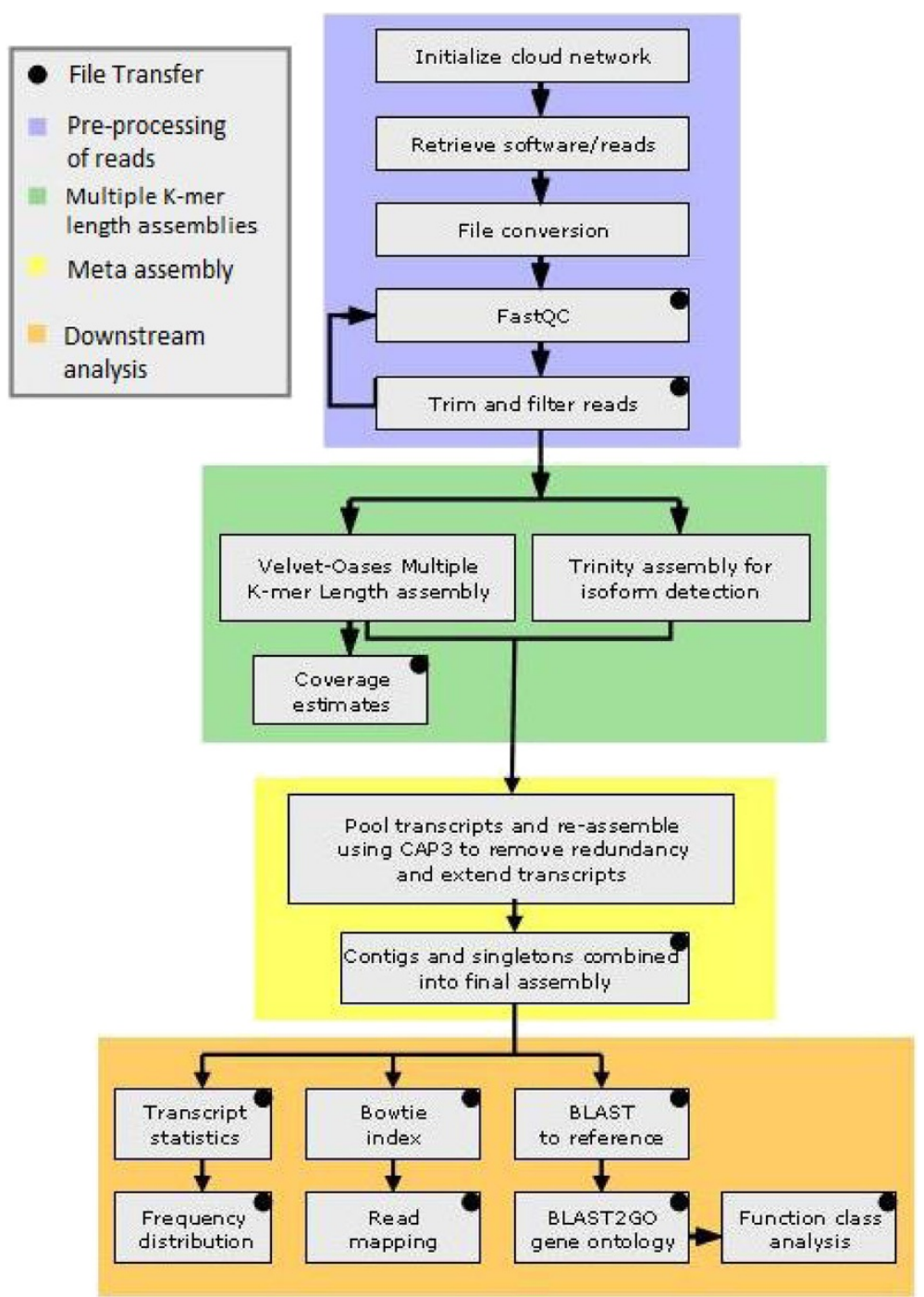

Figure 1 Flowchart of the bioinformatic pipeline. The pipeline performs multiple operations from sequence editing to annotation. First, a cloud network is initialized and algorithms are retrieved and installed. The sequence reads are parsed and filtered for quality and removal of adaptor sequences (blue). Next, assemblies are generated using various k-mer lengths and algorithms to create a diversity of transcript fragments (green). Then, the transcripts from all assemblies are pooled and re-assembled to remove redundant contigs and extend sequences based on overlap (yellow). The resulting multiple k-mer length meta-assembly is then analyzed and formatted for various downstream applications. Reads are mapped back to contigs, genes are annotated, and gene ontology is applied using BLAST and Blast2GO (orange). The pipeline generates an analysis of the assembly and the quantity and distribution of sequences. The resulting data is packaged in an archive for transfer and the cloud network is disbanded.

high memory requirements of Velvet-Oases assembly of 454 sequence reads. An instance with 64 gigabytes (GB) of available memory was used to during initial analysis of assembly performance at different $\mathrm{k}$-mer lengths. This was sufficient to produce assemblies with a k-mer length up to $31 \mathrm{bp}$ after which available memory became a limiting factor, which coincided with a reduction in assembly quality. At the time of this writing high-memory instance types with up to 244GB of available memory are available for larger data sets. Instances were initialized using a publically available Linux operating system disc image hosted by
Amazon. Software, data, and scripts are stored on EBS volumes and software installation is simplified by a script that unpacks and installs all of the packages required for this pipeline to a newly created 'bare' cloud instance. All functional aspects of the pipeline shown in Figure 1 are performed by a wrapper script which sequentially performs the assembly and analysis of sequence data before storing it remotely and terminating the instance to minimize computing cost which is calculated in hourly blocks based on instance type. The pipeline ran to completion in approximately 20 hours. Larger sequence data sets requiring more 
memory and computing time may benefit from separating memory-intensive assembly from processor-intensive downstream analysis as the cost of processing with cloud computing is much lower than reserving large blocks of memory and storage space.

\section{Trimming and quality filtering reads}

Prior to assembly, the reads are processed to remove adaptor sequences, low-quality reads and regions, and highly redundant sequences. The initial quality of the untrimmed sequence reads is assessed using FastQC, which also generates a list of over-represented sequences which may then be removed [37]. The raw sequence reads are then converted to a standard format which is passed on to the FastX Toolkit which removes adaptor sequences using trimming and clipping functions [38]. The reads are subsequently run through the FastX quality filter which removes reads that fail to pass a quality check $(80 \%$ of the bases having a Phred score of 20 or higher, corresponding to a 1:100 base-calling error rate were used for the data presented here). The remaining reads are analyzed for redundancy by FastX and then collapsed into a single representative read. This removes large numbers of identical reads that may result from the amplification process prior to sequencing. Reducing the number of reads can dramatically reduce the amount of memory needed during the assembly process. It can also significantly reduce the amount of time required for assembly, which is an important consideration when generating multiple assemblies [39].

\section{Assembly}

It has been shown that performance varies significantly between assemblers and data sets [40]. This has prompted the development of a number of techniques, such as multiple-k approaches, to retrieve more contigs from the initial sequence reads $[25,41-44]$.

To assemble the $T$. biloba sequence reads we have used a multiple k-mer length approach that creates a large number of assemblies, each of which contains potentially unique transcripts. Because many assembly programs can support multiple k-mer assembly after the addition of custom scripts, we compared the performance of four different assembly programs: Abyss, Newbler, Trinity and Velvet-Oasis, using a previously described protocol (Additional file 1: Table S1) [26,27,40,45-48]. T. biloba sequence reads from multiple life stages were pooled and assembled with a k-mer length of 25 using each of the four assembly programs (Table 1 ). The resulting transcripts were then aligned to the $D$. melanogaster transcriptome. A conservative cut-off value with a minimum aligned length of $400 \mathrm{bp}$ was used to create the distribution in Table 1. While Velvet-Oases produced the longest contigs, Trinity generated a larger number of contigs. A
Table 1 Comparison of assemblers and identification of unique transcripts

\begin{tabular}{lcccc}
\hline Assembler & Contigs & Contig n50 & BLAST hits & Unique hits \\
\hline Velvet-Oases & 18960 & 296 & 5114 & 1817 \\
\hline Abyss & 19664 & 127 & 5341 & 1566 \\
\hline Newbler & 13398 & 208 & 4302 & 1509 \\
\hline Trinity & 25144 & 244 & 6826 & 2194 \\
\hline
\end{tabular}

nucleotide BLAST of contigs in each assembly showed an increase in the number of contigs unique to one assembly in those produced by Trinity and Velvet-Oases. Based on these results, Velvet-Oases was selected for the length of the resulting transcripts and the ease of generating assemblies of different k-mer lengths, and a single Trinity assembly is included to provide isoform detection. The Velvet-Oases and Trinity de novo assembler algorithms have complementary strengths and weaknesses when comparing memory requirements and run-time.

The $T$. biloba sequence data was used to generate assemblies with k-mer lengths of 17, 19, 21, 23, 25, 27, 29, and 31 base pairs. To demonstrate that assemblies with different k-mer lengths recover unique transcripts, the stand-alone BLAST algorithm was used to align contigs from each assembly to a pool of contigs from all assemblies, with the resulting unaligned contigs representing those unique to one assembly (Figure 2). For example, to determine the number of contigs unique to the K17 assembly, the K17 contigs were blasted against the pooled contigs from all other assemblies. If a contig did not align, then it was unique to the $\mathrm{k} 17$ assembly. Contigs were discarded that were less than 200 base pairs. Next, BLAST was performed against D. melanogaster to annotate the unique contigs, and only those contigs with orthology to D. melanogaster were reported (Table 2). After the initial analysis, the pooled assemblies were also annotated using the $D$. melanogaster transcriptome to generate a total number of transcripts for the pool, to which the number of unique transcripts could be compared (Table 2). A significant number of transcripts were represented in only one of the single k-mer length assemblies (Table 2). In total, 2,296 transcripts were identified as unique to a specific assembly using BLAST analysis. For k-mer lengths 17-27, unique transcripts were approximately $2 \%$ of each assembly, and this percentage did not decrease with increasing k-mer length. However, at K29, unique transcripts decreased to only $0.8 \%$ of the total. The number of unique transcripts generated from this analysis is a low estimate because it contains only conserved Drosophila orthologs, and excludes transcripts unique to T. biloba and those too divergent to be identified by BLAST. Therefore, the number of unique transcripts recovered from different k-mer assemblies is likely higher. Our analysis confirms that restricting 


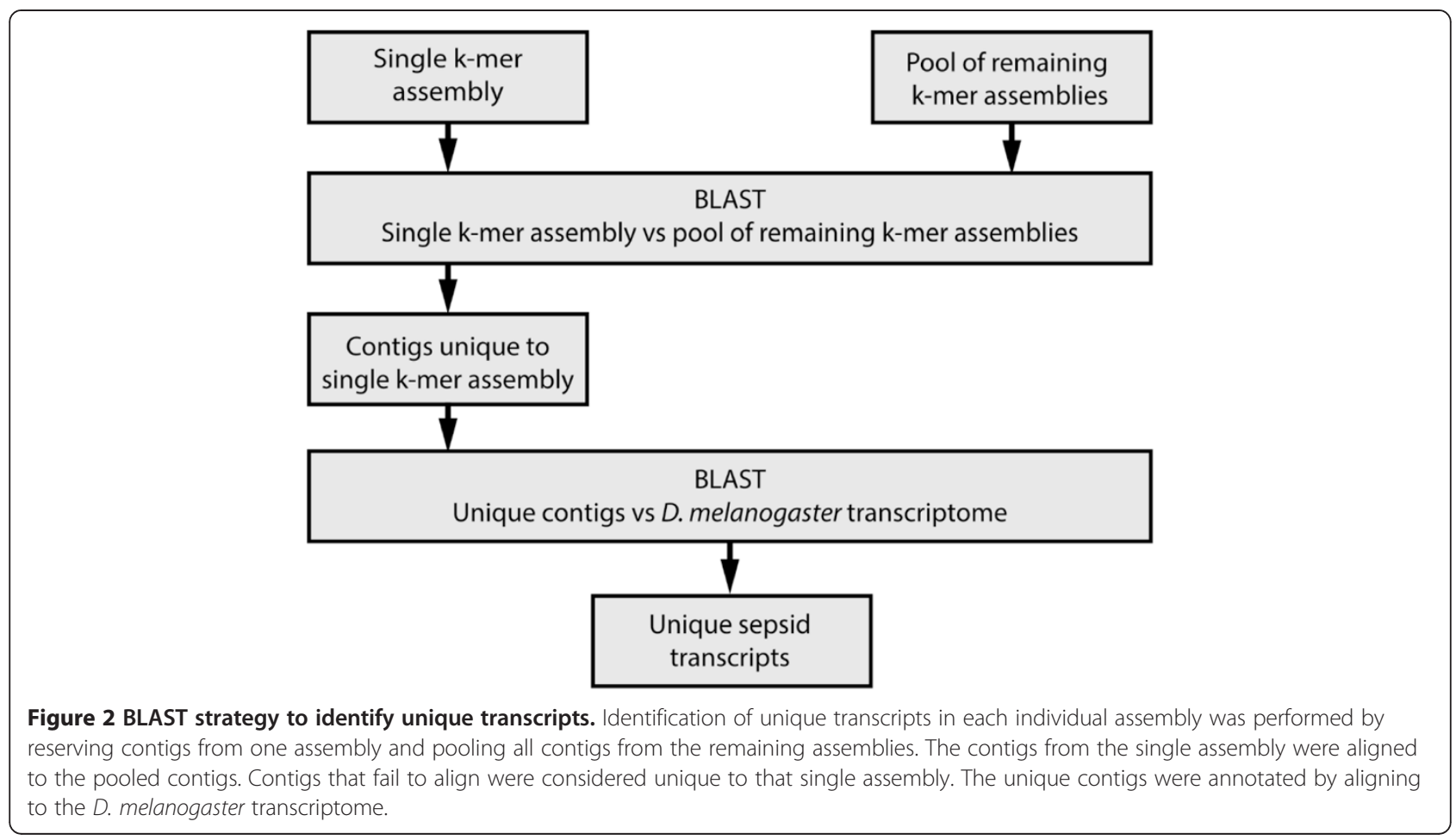

assemblies to only a single k-mer length limits the number of transcripts recovered, regardless of which $\mathrm{k}$-mer length is chosen.

\section{Meta-assembly}

The assemblies generated with k-mer lengths of 23, 25, 27 , and 29 base pairs were combined through metaassembly which extends contigs found in multiple assemblies and retains contigs found in only one. K-mer lengths shorter than 23 resulted in a large number of singletons and short contigs. Assemblies with a k-mer length larger than 29 required much larger memory allocations and computational time and were more conservative than other assemblies resulting in diminishing returns in which

Table 2 Unique transcripts per k-mer length in pairedend assemblies using Velvet-Oases

\begin{tabular}{lcccc}
\hline $\begin{array}{l}\text { K-mer } \\
\text { length }\end{array}$ & $\begin{array}{c}\text { Total } \\
\text { transcripts }\end{array}$ & $\begin{array}{c}\text { Transcripts absent } \\
\text { from one or } \\
\text { more assemblies }\end{array}$ & $\begin{array}{c}\text { Transcripts } \\
\text { unique to } \\
\text { one assembly }\end{array}$ & $\begin{array}{c}\text { \% unique } \\
\text { transcripts }\end{array}$ \\
\hline 17 & 21296 & 2331 & 464 & 2.2 \\
\hline 19 & 20080 & 2105 & 397 & 2.0 \\
\hline 21 & 17950 & 1875 & 410 & 2.3 \\
\hline 23 & 16668 & 1686 & 316 & 1.9 \\
\hline 25 & 15894 & 1434 & 280 & 1.8 \\
\hline 27 & 15496 & 2398 & 313 & 2.0 \\
\hline 29 & 15138 & 3855 & 116 & 0.8 \\
\hline Total & 122522 & 15684 & 2296 & 1.9 \\
\hline
\end{tabular}

larger k-mer word sizes produce few novel transcripts not present in other assemblies.

The CAP3 software was used to construct the metaassembly [28]. The CAP3 software removes the redundancy generated within and between assemblies of different k-mer lengths to consolidate the transcripts. Consolidating the results of all k-mer assemblies created a pool of 138,954 contigs. CAP3 clustered and assembled these sequences into a meta-assembly of 15,984 extended contigs and 8,511 singletons. The singletons represent sequences for which no overlap exists between assemblies and thus could not be extended by CAP3. The final meta-assembly consisted of 24,495 contigs with a mean sequence length 1,403 base pairs, an increase of $372 \mathrm{bp}$ (34.1\%) compared to the K25 assembly.

Analysis of transcript length revealed that the total number of base pairs assembled improved significantly from 17.4 $\mathrm{Mb}$ to $32.7 \mathrm{Mb}$ and the mean contig length increased by $310 \mathrm{bp}$ from $1,093 \mathrm{bp}$ to $1,403 \mathrm{bp}$. A frequency distribution of the number of contigs of a given length (Figure 3) shows an increase in the number of longer contigs in the meta-assembly, compared to the single k-mer assemblies and the Trinity assembly. The single k-mer assemblies have a relatively high number of singletons (sequences of less than $500 \mathrm{bp}$ ). The number of singletons was greatly reduced in the meta-assembly, indicating that meta-assembly was able to extend contigs by incorporating singletons. To demonstrate that contigs from different k-mer assemblies were used to 


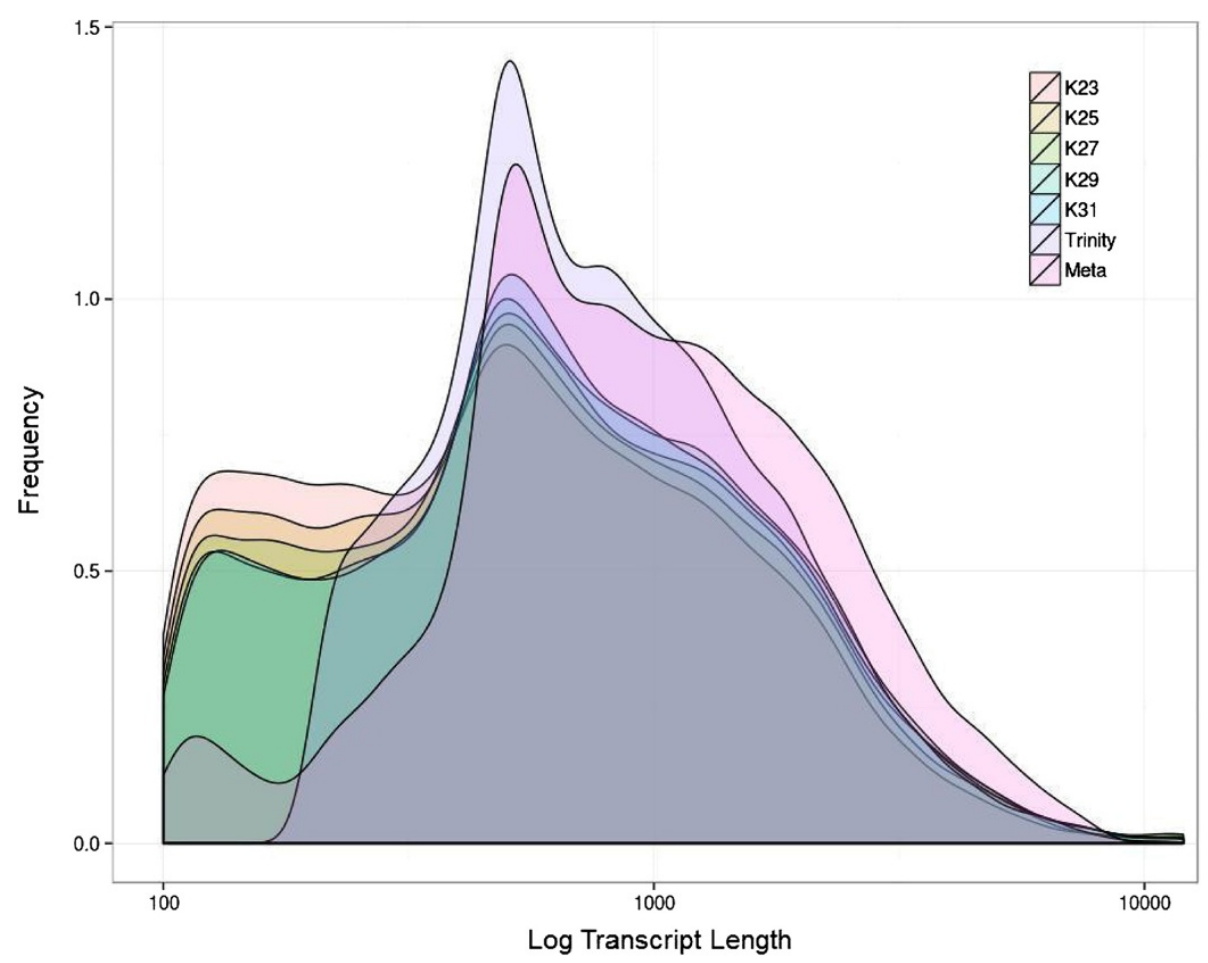

Figure 3 Frequency distribution of transcript lengths by assembly. A plot of the quantity of transcripts with a given length per assembly shows differences in assembly output and a pronounced peak representing the median transcript length. The meta-assembly was generated by the re-assembly of all k-mer lengths using CAP3. Meta-assembly improved transcript length, as indicated by the leading edge of the graph. Meta-assembly also reduced the number of short contigs, compared to the single k-mer assemblies. Trinity automatically removes contigs smaller than 200 base pairs.

create extended consensus contigs, genes from a candidate list of transcription factors were tracked from the 454 reads through the assembly and meta-assembly process (Table 3). Transcription factors are generally low abundance transcripts, and therefore full-length sequences are less likely to be recovered in single k-mer assemblies. Five out of the seven transcripts were extended through CAP3 re-assembly (Table 3). Primers were designed for four sequences and PCR amplification using T. biloba cDNA produced bands of the expected size, indicating that these extended contigs are correctly assembled transcripts (Additional file 2: Figure S1). To better visualize how

Table 3 Transcripts of interest extended by meta-assembly

\begin{tabular}{lcc}
\hline Identity & Meta-assembly & Individual assembly \\
\hline Engrailed* & 1140 & 1140 \\
\hline Escargot* $^{*}$ & 1244 & 782 \\
\hline Evenskipped $^{*}$ & 876 & 717 \\
\hline Extradenticle & 1143 & $574,417,138$ \\
\hline Hunchback & 800 & 699,472 \\
\hline Sex-combs reduced & 232 & 281 \\
\hline Ultrabithorax & 1084 & $526,368,370,874$ \\
\hline *Validated by PCR & & \\
\hline
\end{tabular}

meta-assembly extends transcript length, we examined in further detail how extradenticle contigs from different assemblies were meta-assembled (Figure 4). The meta-assembly recovered the entire length of the coding sequence of the Tbil-exd transcript, as compared to Drosophila. Assembling the full transcript required contigs from multiple assemblies, and only a subset of the individual assemblies contained sequences fragments for the middle of the transcript. Contigs from assemblies outside the 23-29 k-mer range show a reduction in coverage caused by fragmentation in assemblies with shorter k-mer lengths and conservative assembly with larger k-mer lengths. The Tbil-exd sequence contains several single nucleotide insertions within the region aligned to the Drosophila reference and $83 \%$ of the nucleotide identities are conserved.

To determine whether meta-assembly would improve transcriptome quality across taxa, the meta-assembly process was performed on three archived datasets (Oncopeltus fasciatus: SRR057573; Silene vulgaris: SRR245489; Ictidomys tridecemlineatus: SRR352220) using the same pipeline used to generate the T. biloba transcriptome. (Table 4; Figure 5). The meta-assemblies for each of the four datasets were compared to a single $25 \mathrm{k}$-mer length assembly. 


\section{T. biloba transcripts were extended by meta-assembly}

\section{T. biloba extradenticle coding sequence}

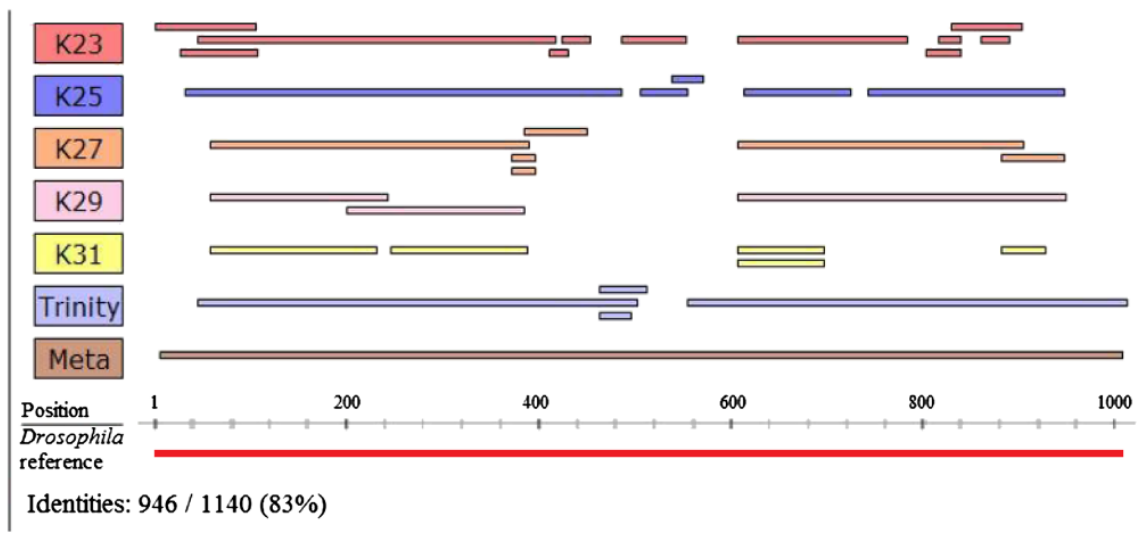

Figure 4 Extension of extradenticle sequence by meta-assembly. Contigs generated by multiple k-mer lengths were consolidated by meta-assembly to recover the entire coding sequence of the gene extradenticle from sequence fragments. Contigs from individual assemblies of multiple k-mer lengths are shown in alignment to the meta-assembly and the Drosophila transcript. The k-mer length 31 contigs were not included in the meta-assembly and show a reduction in coverage compared to other assemblies. Assemblies with shorter k-mer lengths also show a reduction in coverage but are not shown due to excessive fragmentation which results in a large number of short contigs that cannot be confidently aligned. The extended transcript aligns to the full length of the Drosophila reference sequence with 83\% nucleotide sequence conservation.

We used multiple metrics to compare transcription quality between the $25 \mathrm{k}$-mer length assembly and the meta-assembly including: number of base pairs assembled, number of contigs, percent of reads used in the contigs, and median contig length (Figure 5; Table 4). In all four datasets, the number of base pairs assembled was greater in the meta-assembly. The greatest increased was observed in I. tridecemlineatus in which the number of base pairs assembled doubled with meta-assembly. Overall, the total number of assembled base pairs is $60.1 \%$ to $105.6 \%$ greater. The increase in base-pairs assembled was mirrored by an increase in contig length in all four species, as measured by mean contig length, median contig length, and n50 (Figure 5D; Table 4). The increase in length is presumably a result of incorporating more reads, because the percent of total reads that were assembled into contigs also increased with metaassembly (Figure 5B). In addition to increasing contig length, the meta-assembly also increased contig number in the I. tridecemlineatus, S. vulgaris, and O. faciatus, data sets (Figure $5 \mathrm{~B}$ ). The increase in contig number is further evidence that meta-assembly recovers unique contigs from different $\mathrm{k}$-mer length assemblies. The gain in contig number was likely even greater than the observed increase because the $25 \mathrm{k}$-mer assembly includes redundant contigs, whereas the meta-assembly does not. The same pre-processing steps were used to generate the filtered reads for both the $25 \mathrm{k}$-mer and meta-

Table 4 Single and multiple k-mer length meta assembly across 4 species

\begin{tabular}{|c|c|c|c|c|c|c|}
\hline Assembly & Base-pairs & $\mathbf{n}$ & Median & Mean & n50 & $\%$ reads used \\
\hline \multicolumn{7}{|l|}{ I. tridecemlineatus } \\
\hline 25-mer assembly & 25446725 & 33363 & 460 & 762 & 1328 & $49.97 \%$ \\
\hline Meta assembly & 52328097 & 50869 & 608 & 1028 & 1708 & $70.26 \%$ \\
\hline \multicolumn{7}{|l|}{ S. vulgaris } \\
\hline 25-mer assembly & 21706584 & 34262 & 404 & 633 & 1124 & $66.31 \%$ \\
\hline Meta assembly & 40068740 & 43475 & 815 & 921 & 1351 & $85.45 \%$ \\
\hline \multicolumn{7}{|l|}{ O. fasciatus } \\
\hline 25-mer assembly & 9487925 & 15886 & 421 & 597 & 894 & $69.12 \%$ \\
\hline Meta assembly & 18283749 & 18106 & 797 & 1009 & 1312 & $81.80 \%$ \\
\hline \multicolumn{7}{|l|}{ T. biloba } \\
\hline 25-mer assembly & 20431185 & 22423 & 549 & 911 & 1571 & $58.87 \%$ \\
\hline Meta assembly & 32887248 & 24495 & 887 & 1342 & 2010 & $64.01 \%$ \\
\hline
\end{tabular}


A

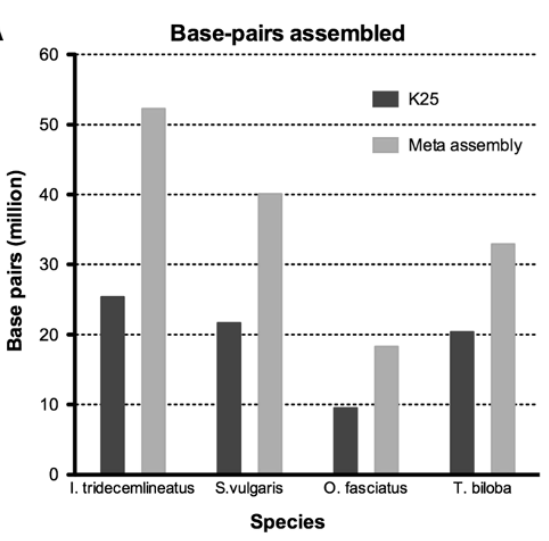

C

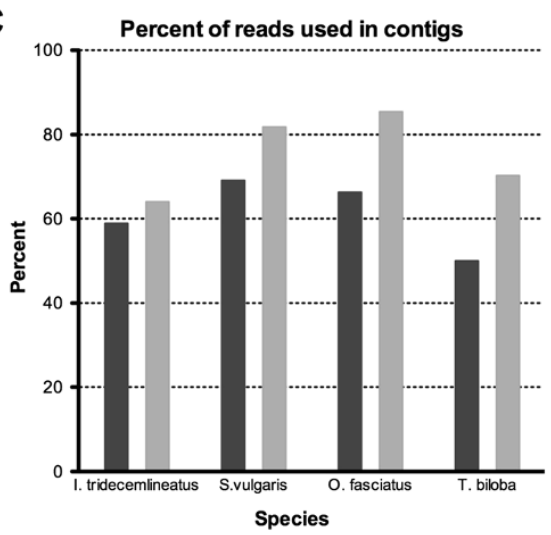

B

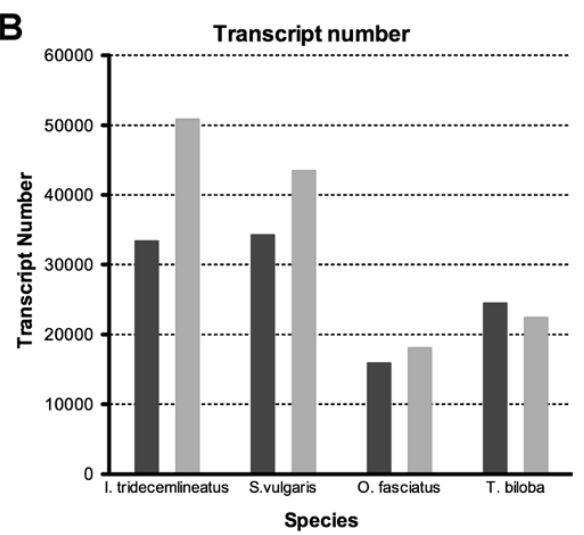

D

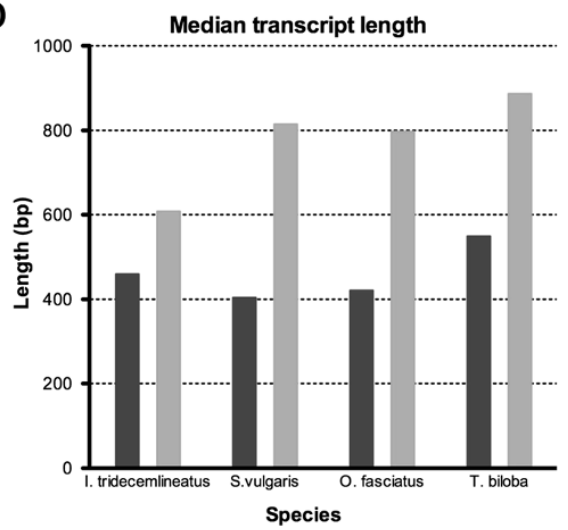

Figure 5 Performance of meta-assembly across species. A single assembly using Velvet-Oases with a K-mer length of 25 (light gray) was compared to the multiple k-mer length meta-assembly (black) for four species. Meta-assembly improved overall transcript length. The total assembled base-pairs (A), transcript number (B), percent of reads used in contigs (C), and median transcript length (D) show improvement in transcript assembly.

assemblies but the $25 \mathrm{k}$-mer assemblies did not undergo a secondary assembly to remove internal redundancy. When applied to a single Velvet-Oases assembly, CAP3 reduces the number of contigs by $5.5 \%$. The only species to see a reduction in the number of contigs after metaassembly was $T$. biloba. We hypothesize this reduction was due to either elimination of duplicates, consolidation of contigs, or both.

\section{Alignment and annotation of the Themira biloba transcriptome}

The $T$. biloba transcriptome was annotated using the $D$. melanogaster transcriptome as a reference. The pipeline aligned the $T$. biloba transcripts to $D$. melanogaster using the standalone BLAST package and a reference database available from FlyBase [49]. 11,008 transcripts from the meta-assembly were identified via BLAST as homologous to Drosophila sequences (44.9\%). We found that the aligned $T$. biloba sequences were $82.3 \%$ conserved (mean sequence conservation taken from a subset of 500 BLAST hits) indicating that BLAST may not be sufficient to identify some sequences. Therefore, sequence divergence between the two species could explain why over half the T. biloba contigs in the meta-assembly could be annotated based on Drosophila. However, contig mis-assembly could also cause low annotation rates. To determine whether sequence divergence or mis-assembly was the cause, we annotated the $\mathrm{T}$. biloba transcriptome with a more closely related Dipteran.

Sepsidae is more closely related to Tephritidae than the drosophilids [17], so it would be expected that higher sequence conservation exists between these two families, and that comparison to a tephritid would identify more transcripts. To determine whether such a comparison would identify more transcripts than Drosophila, a transcriptome was constructed using archived Illumina sequence reads from adult male and female Bactrocera dorsalis (SRR818498, SRR818496) [50]. Bi-directional alignments were created using T. biloba, B. dorsalis, and D. melanogaster. Contrary to our prediction, the alignments between $T$. biloba and $B$. dorsalis did not show increased aligned contigs or even conserved sequence versus Drosophila (Table 5). On average, B. dorsalis had around the same sequence similarity to $T$. biloba that 
Table 5 BLAST matches and percent identities

\begin{tabular}{|c|c|c|c|c|c|}
\hline Query & Database & Matched & Unmatched & Mean length & Mean $\%$ conserved \\
\hline T. biloba & D. melanogaster & 11008 & 13487 & 1200 & $82.21 \%$ \\
\hline T. biloba & B. dorsalis & 6273 & 18222 & 729 & $82.46 \%$ \\
\hline B. dorsalis & D. melanogaster & 9334 & 41053 & 802 & $84.17 \%$ \\
\hline B. dorsalis & T. biloba & 6277 & 40273 & 726 & $85.99 \%$ \\
\hline D. melanogaster & T. biloba & 13544 & 23852 & 1336 & $82.37 \%$ \\
\hline
\end{tabular}

Drosophila did, and the number of matching transcripts actually decreased, as did the average length of the matching region. The decrease in number of matches may be due to the nature of the datasets. The Drosophila transcriptome includes multiple life stages and has a high level of coverage, whereas the $B$. dorsalis transcriptome only includes the adult stage [50]. Decreased representation could result in alignment of fewer genes even though the amount of sequence divergence is similar. In the end, annotation to $B$. dorsalis had the same limitations as Drosophila because of sequence divergence in the Sepsidae lineage.

To determine whether comparison with other more complete databases could increase the number of annotated contigs, the contigs from the T. biloba meta-assembly were compared to the SwissProt databases. SwissProt has the ability to compare translated contigs, thus reducing the problem posed by nucleotide divergence. Additional transcripts were annotated through BLASTx against the SwissProt database, which had not been annotated through the comparison with D. melanogaster. An expectvalue cutoff of 0.00001 resulted in alignment of 16,705 (68.2\%) of the translated sequences to sequences in the SwissProt database, which was a difference of 5,697 contigs (23.2\%) compared to nucleotide BLAST against a single species. Analysis was performed to determine known protein domains in the Pfam database using the Trinity utility TransDecoder [51]. An additional 221 contigs that had not been annotated were found to contain Pfam domains increasing the number of contigs identified by at least one searched database to 16,926 (69.1\%). The number of annotated contigs compares favorably to other de novo assemblies [52-54]. The high percentage of annotated transcripts indicates that the contigs generated through meta-assembly are true transcripts, and not mis-assembled contigs. Further improvements in annotation likely require greater coverage through increased sequencing depth and a larger sequence data set.

To determine ontology, T. biloba transcripts were submitted for KEGG pathway analysis resulting in 5,080 contigs with identified functions. Many developmentally import pathways involved in cell signaling such as the notch pathway were near complete (Additional file 3: Table S2). Transcripts were assigned gene ontologies, which were then grouped by function (Figure 6) to determine whether the transcripts recovered from the meta-assembly were representative of the main cellular processes. A broad range of functional groups were present in the assembly, indicating that transcripts representing many different kinds of proteins were recovered. The distribution of contig gene ontologies is similar to those found in the distribution of GO terms found in the Drosophila transcriptome and other de novo transcriptome assembly efforts $[34,55,52,54]$.

\section{Discussion}

\section{Bioinformatics and data management}

The de novo assembly of a transcriptome presents multiple challenges including computational requirements and accurate assembly of low abundance transcripts. Here we present a pipeline for de novo assembly that uses cloud computing and a multiple k-mer meta-assembly processes. The strength of a distributed, cloud-based approach to transcriptome assembly and sequence analysis is its versatility and the low initial investment in data processing $[23,56]$. We have found the primary advantage of hosting data analysis off-site is the ability to construct a low-cost, scalable network on demand with unrestricted access. The increased computing power is particularly important when generating multiple de novo assemblies, as is done in our meta-assembly processes. Meta-assembly processes that use a multiple k-mer length approach have been previously demonstrated to significantly improve the quality of transcriptomes [24,57].

The pipeline presented here incorporates an extensive and automated toolkit for parsing and trimming sequence reads prior to multiple k-mer assembly and the generation of a meta-assembly that best represents the transcripts available to be recovered. Automated sequence analysis tools are included to provide graphical views of read quality, transcript length and coverage per assembly, transcript extension, annotation information of sequence homologs from various databases, and the presence of unique sequences, and the assembly parameters used to recover the sequences.

\section{Increasing transcriptome quality with meta-assembly}

We validated our pipeline by assembling three previous published transcriptomes and the transcriptome of the 


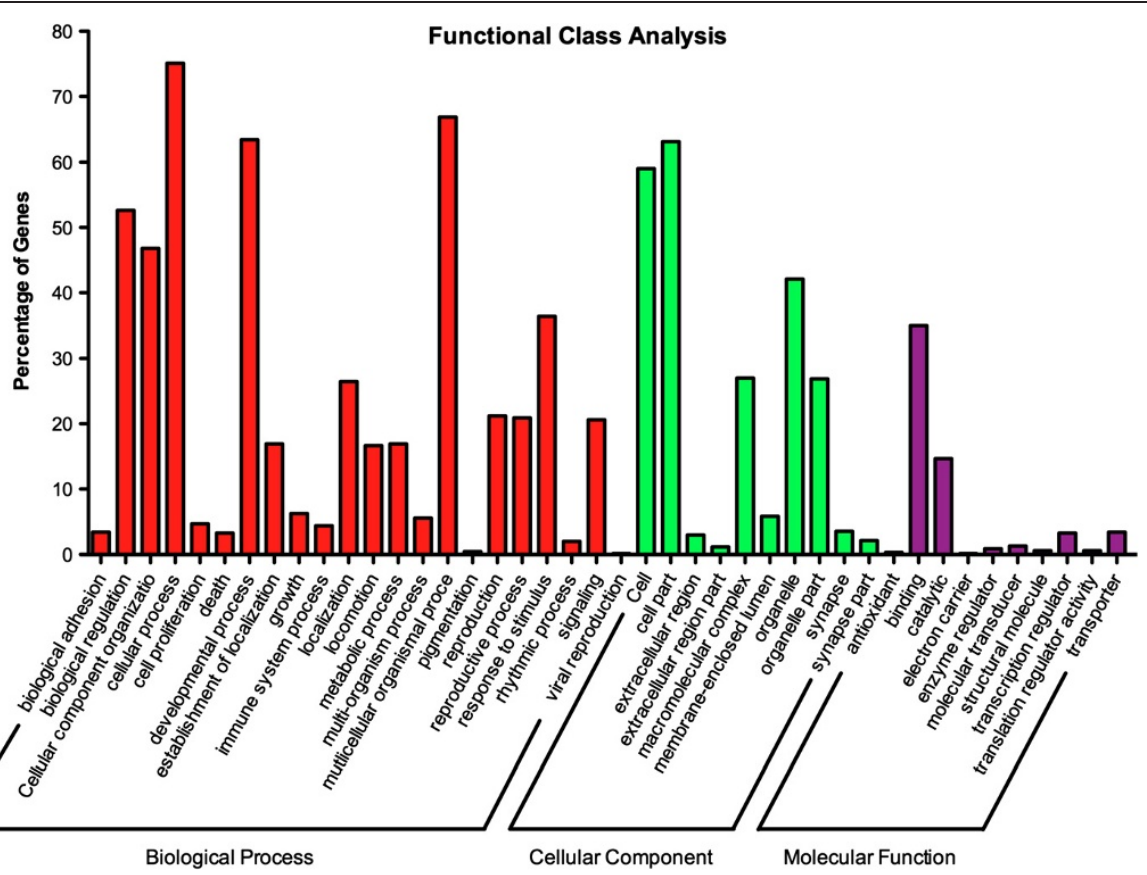

Figure 6 Gene Ontology classification of the T. biloba transcriptome. Gene Ontology (GO) was assigned to all contigs from the T. biloba meta-assembly. Gene ontologies were group into three main categories and 42 sub-categories. Contigs are grouped by the percentage of sequences that match a specific GO term within three major groups. The most abundant transcripts represent the sub-categories containing structural proteins and regulators of various cellular processes.

sepsid fly T. biloba, which was sequenced as part of this project. Transcriptome quality was compared between our pipleline, which employs a meta-assembly process, and the standard practice of using a single $25 \mathrm{bp}$ k-mer length for assembly. In all four species, the metaassembly increased the number of base pairs assembled, increased the length of contigs, increased the percentage of reads used in the contigs and recovered a greater number of transcripts than the $25 \mathrm{k}$-mer assembly. The increased quality of meta-assembly was further investigated in the T. biloba transcriptome by tracking the improvement in a candidate list of low abundance transcripts. For a subset of these transcripts, RT-PCR confirmed that meta-assembly increased the length of the transcripts by connecting fragments recovered from multiple k-mer length assemblies.

\section{Conclusions}

We have assembled transcript sequences from the complete life cycle of T. biloba, a sepsid fly which exhibits primary gain of a novel trait, and identified many developmentally important genes. These transcripts represent the first large-scale sequencing that has been performed within the family Sepsidae, a large and diverse family with over 250 species distributed globally. Sepsid flies have been used for taxonomic and behavioral studies and have diverse genital and appendage morphologies, but lack of sequence data has made genetic investigation of these traits difficult $[58,9,4,8,11,2]$. While many orthologous genes retain their functions between dipterans, large regions of gene sequence are often not conserved $[18,59]$.

The T. biloba transcriptome and many of the genes we have identified will be used for future RNA-Seq studies of comparative gene expression, knockdown, and in situ hybridization experiments. Sequence for many developmentally important genes and transcription factors of interest were obtained including members of the HOX family and those associated with embryonic and morphological development. In addition, many sequences for genes involved in cell signaling pathways such as notch and torso signaling were recovered. Sequence for the $T$. biloba doublesex ortholog as well as several transcripts associated with mating and courtship in Drosophila were also recovered which aids investigation of the sepsid sex allocation pathway and the genetic mechanisms behind behavioral traits associated with the sepsid novel appendage.

As more genomes become available, researchers using non-model organisms will have the opportunity to assemble RNA-seq reads to reference genomes of closely related species. Assembling to a reference, when available, yields a higher quality transcriptome than de novo assembly, and this result is robust to low-levels of genomic divergence between species $[42,44]$. Although these findings are encouraging, those working with non-model organisms should proceed with caution [60]. Based on in silico studies, 
assembling to a reference that has a sequence divergence greater than $15 \%$ decreases the number of transcripts recovered compared to de novo assembly [44]. In our case, assembling the $T$. biloba reads to the Drosophila genome would have been inappropriate because the $17 \%$ sequence divergence between the two species would have resulted in decreased transcript recovery compared to de novo assembly. Choosing a closer relative based on phylogeny does not necessarily solve the problem, as our additional comparison to $B$. dorsalis revealed. Because the amount of sequence divergence between a non-model organism and its closely related reference species is rarely known prior to high-throughput sequencing, de novo assembly remains a powerful tool for recovering transcripts in non-model organisms.

\section{Methods}

\section{T. biloba colony}

Cultures of T. biloba were maintained in an incubator at $25 \mathrm{C}$ with a 16:8 hour light-dark cycle in overlapping generations. Larvae were raised in Petri dishes and fed agar mixed with soy infant formula (ProSobee) covered with a $1.0 \mathrm{~cm}$ layer of cow dung. Adults were fed honey mixed with water and provided with cow dung to facilitate mating and egg-laying.

\section{Tissue collection}

Tissue was collected from embryos, $3^{\text {rd }}$ instar larva, and 48-72 hour pupa. During collection all material was stored at $-80^{\circ} \mathrm{C}$ in RNALater, prior to shipment to the sequencing facility. Embryos were collected regularly and washed several times with an egg wash solution of $0.12 \mathrm{M} \mathrm{NaCl}$ and $0.01 \%$ Triton X-100 to remove dung. The eggs were dechorionated using a $3 \%$ bleach solution. Third instar, wandering-phase larvae were everted in PEM buffer (100 mM PIPES-disodium salt, $2.0 \mathrm{mM}$ EGTA, $1.0 \mathrm{mM} \mathrm{MgSO}_{4}$ anhydrous, $\mathrm{pH}$ 7.0) to facilitate RNA extraction. Prior to pupation, gut-purged larvae were allowed to wander on moistened filter paper to remove dung and particulates. Pupae were staged to 48-72 hours before collection. All samples were stored in RNALater overnight at $4^{\circ} \mathrm{C}$ and transferred to $-80^{\circ} \mathrm{C}$ for storage prior to sequencing.

\section{Sequencing}

RNA isolation, library cDNA preparation, and 454 sequencing were performed by the University of Arizona Genetics Core (UAGC). Prior to sequencing, the cDNA was screened using a 2100 Bioanalyzer (Agilent Technologies). Sequencing was done on a GS FLX Titanium (454 Life Sciences). Embryos, larvae, and pupae were sequenced separately, creating 3 separate pools of sequence. Approximately 1.48 million reads total with an average length of $400 \mathrm{bp}$ were generated.

\section{Assembly and annotation}

Pre-processing of the sequence reads generated from $T$. biloba was performed using the FastX Toolkit [38]. Adaptor sequences were removed using the trimmer function. The quality filter removed sequences in which $80 \%$ of the base pairs had a Phred score of less than 20. The remaining 1.01 million reads were then converted to FASTA. The FastX collapsing tool was used to consolidate redundant sequences to reduce the amount of memory needed during the assembly process. An assembly was performed using the collapsed reads to determine the reduction in memory required for assembly (Additional file 4: Figure S2). We determined that although collapsing the reads significantly reduced the memory requirements for assembly, it was not necessary for the data sets described in this publication and may lead to a reduction in coverage. FastQC (v0.10.1) was used to assess the quality of reads before and after pre-processing [37].

Paired-end assemblies with K-mer lengths of 19 to 29 were generated using Velvet-Oases with an insert size of $200 \mathrm{bp}[26,27]$. Trinity was used to generate an additional paired-end assembly $[47,48]$. The resulting contigs were aligned to Drosophila using standalone BLAST to identify developmentally important transcripts. A BLAST alignment was then performed using each individual assembly as the query and the pooled contigs from all other assemblies as the database to identify contigs unique to each assembly. The assemblies were then concatenated and the pool of 138,954 transcripts was reassembled using CAP3 [28].

\section{Additional files}

The 454 reads from this study have been archived at the NCBI Sequence Read Archive under BioProject [PRJNA:218740]. Custom scripts for assembly and analysis of the T. biloba transcriptome and a disc image of the complete pipeline with all programs and scripts used in this pipeline is available at https://sourceforge.net/projects/ themiratranscriptome.

\section{Additional file 1: FastQC reports for untrimmed and trimmed sequence reads. Quality reports generated before and after quality filtering and trimming show an improvement in multiple quality metrics. \\ Additional file 2: PCR validation of assembled contigs. Primers designed from bioinformatically generated contigs annotated using the Drosophila transcriptome produced the expected band sizes (from left to right) for engrailed, escargot, and evenskipped. \\ Additional file 3: KEGG classification and functional maps of assembled contigs. Contigs annotated using KEGG Automatic \\ Annotation Server identified sequences in a broad range of functional groups including developmental pathways and cell signaling.}

Additional file 4: Average distribution of coverage of T. biloba contigs. Coverage estimates were generated using the Velvet software.

Competing interests

The authors declare that they have no competing interests. 


\section{Authors' contributions}

DM, JB, and AT designed the research plan. DM and AT collected tissue and isolated RNA. DM assembled and analyzed the data and drafted the manuscript. AT maintained the animals, selected the additional sequence data sets, and helped analyze the data. JB helped write the manuscript, and obtained funding for the research. ID provided bioinformatic training and consultation. All authors contributed to and approve the content of the final manuscript.

\section{Acknowledgements}

We would like to thank George Yocum, Joe Rinehart, Bill Kemp, Jennifer Momsen, Erika Offerdahl, Stuart Haring and the members of the Bowsher lab for their discussion and feedback of the research plan, pipeline, and results. We thank the members of the Dworkin lab for hosting us and helping us identify bioinformatic training and tutorial resources. We thank Rudolf Meier of the National University of Singapore for providing us with the animal colony, and for discussion of sepsid taxonomy. We would like to thank the North Dakota State University College of Science and Mathematics, the Department of Biological Sciences, and the EDEN Research Coordination Network and the National Science Foundation (HRD-0811239) for their financial contributions.

\section{Author details}

'Department of Biological Sciences, North Dakota State University, 1340 Bolley Drive, 218 Stevens Hall, Fargo, ND 58102, USA. ²Department of Zoology, Michigan State University, 328 Giltner Hall, East Lansing, MI 48823, USA

\section{Received: 4 November 2013 Accepted: 3 March 2014}

Published: 12 March 2014

\section{References}

1. Pont AC: The Sepsidae (Diptera) of Europe. Leiden; Boston: Brill: [Fauna Entomologica Scandinavica, v. 37]; 2002.

2. Bowsher JH, Ang Y, Ferderer T, Meier R: DECIPHERING THE EVOLUTIONARY HISTORY AND DEVELOPMENTAL MECHANISMS OF A COMPLEX SEXUAL ORNAMENT: THE ABDOMINAL APPENDAGES OF SEPSIDAE (DIPTERA). Evolution 2013, 67:1069-1080

3. Ingram KK, Laamanen T, Puniamoorthy N, Meier R: Lack of morphological coevolution between male forelegs and female wings in Themira (Sepsidae: Diptera: Insecta). Biol J Linn Soc 2008, 93:227-238.

4. Puniamoorthy N, Ismail MRB, Tan DSH, Meier R: From kissing to belly stridulation: comparative analysis reveals surprising diversity, rapid evolution, and much homoplasy in the mating behaviour of 27 species of sepsid flies (Diptera: Sepsidae). J Evol Biol 2009, 22:2146-2156.

5. Baena ML, Eberhard WG: Appearances deceive: female "resistance" behaviour in a sepsid fly is not a test of male ability to hold on. Ethol Ecol Evol 2007, 19:27-50.

6. Martin OY, Hosken DJ: The evolution of reproductive isolation through sexual conflict. Nature 2003 423:979-982.

7. Blanckenhorn WU, Kraushaar URS, Teuschl Y, Reim C: Sexual selection on morphological and physiological traits and fluctuating asymmetry in the black scavenger fly Sepsis cynipsea. J Evol Biol 2004, 17:629-641.

8. Puniamoorthy N, Schäfer MA, Blanckenhorn WU: Sexual selection accounts for the geographic reversal of sexual size dimorphism in the dung fly, sepsis punctum (Diptera: Sepsidae). Evol Int J Org Evol 2012, 66:2117-2126.

9. Puniamoorthy N, Su K, Meier R: Bending for love: losses and gains of sexual dimorphisms are strictly correlated with changes in the mounting position of sepsid flies (Sepsidae: Diptera). BMC Evol Biol 2008, 8:155.

10. Eberhard WG: Multiple origins of a major novelty: moveable abdominal lobes in male sepsid flies (Diptera: Sepsidae), and the question of developmental constraints. Evol Dev 2001, 3:206-222.

11. Eberhard WG: Species-specific genitalic copulatory courtship in sepsid flies (Diptera, Sepsidae, Microsepsis) and theories of genitalic evolution. Evol Int J Org Evol 2001, 55:93-102.

12. Eberhard WG: Sexual behavior and morphology of Themira minor (Diptera: Sepsidae) males and the evolution of male sternal lobes and genitalic surstyli. Can Entomol 2012, 135:569-581.

13. Eberhard WG: Evolutionary Conflicts of Interest: Are Female Sexual Decisions Different? Am Nat 2005, 165:S19-S25.
14. Bowsher JH, Nijhout HF: Evolution of novel abdominal appendages in a sepsid fly from histoblasts, not imaginal discs. Evol Dev 2007, 9:347-354.

15. Bowsher $\mathrm{JH}$, Nijhout HF: Partial co-option of the appendage patterning pathway in the development of abdominal appendages in the sepsid fly Themira biloba. Dev Genes Evol 2009, 219:577-587.

16. Wiegmann BM, Yeates DK, Thorne $J \mathrm{~L}$, Kishino H: Time flies, a new molecular time-scale for brachyceran fly evolution without a clock. Syst Biol 2003, 52:745-756.

17. Wiegmann BM, Trautwein MD, Winkler IS, Barr NB, Kim J-W, Lambkin C, Bertone MA, Cassel BK, Bayless KM, Heimberg AM, Wheeler BM, Peterson KJ, Pape T, Sinclair BJ, Skevington JH, Blagoderov V, Caravas J, Kutty SN, Schmidt-Ott U, Kampmeier GE, Thompson FC, Grimaldi DA, Beckenbach AT, Courtney GW, Friedrich M, Meier R, Yeates DK: Episodic radiations in the fly tree of life. Proc Natl Acad Sci 2011, 108:5690-5695.

18. Hare EE, Peterson BK, lyer VN, Meier R, Eisen MB: Sepsid even-skipped Enhancers Are Functionally Conserved in Drosophila Despite Lack of Sequence Conservation. PLOS Genet 2008, 4:e1000106.

19. Schwarz D, Robertson HM, Feder JL, Varala K, Hudson ME, Ragland GJ, Hahn DA, Berlocher SH: Sympatric ecological speciation meets pyrosequencing: sampling the transcriptome of the apple maggot Rhagoletis pomonella. BMC Genomics 2009, 10:633.

20. Zheng W, Peng T, He W, Zhang H: High-Throughput Sequencing to Reveal Genes Involved in Reproduction and Development in Bactrocera dorsalis (Diptera: Tephritidae). PLOS ONE 2012, 7:e36463.

21. Hsu J-C, Chien T-Y, Hu C-C, Chen M-JM WW-J, Feng H-T, Haymer DS, Chen C-Y: Discovery of Genes Related to Insecticide Resistance in Bactrocera dorsalis by Functional Genomic Analysis of a De Novo Assembled Transcriptome. PLOS ONE 2012, 7:e40950.

22. Nirmala X, Schetelig MF, YU F, Handler AM: An EST database of the Caribbean fruit fly, Anastrepha suspensa (Diptera: Tephritidae). Gene 2013, 517:212-217.

23. Sboner A, Mu XJ, Greenbaum D, Auerbach RK, Gerstein MB: The real cost of sequencing: higher than you think! Genome Biol 2011, 12:125.

24. Surget-Groba Y, Montoya-Burgos Jl: Optimization of de novo transcriptome assembly from next-generation sequencing data. Genome Res 2010, 20:1432-1440.

25. Gruenheit N, Deusch O, Esser C, Becker M, Voelckel C, Lockhart P: Cutoffs and k-mers: implications from a transcriptome study in allopolyploid plants. BMC Genomics 2012, 13:92.

26. Velvet: a sequence assembler for very short reads. [http://www.ebi.ac.uk/ zerbino/velvet/]

27. Oases: a transcriptome assembler for very short reads. [http://www.ebi.ac.uk zerbino/oases/]

28. Huang $X$, Madan A: CAP3: A DNA sequence assembly program. Genome Res $1999,9: 868-877$

29. Conesa A, Götz S, García-Gómez JM, Terol J, Talón M, Robles M: Blast2GO: a universal tool for annotation, visualization and analysis in functional genomics research. Bioinforma Oxf Engl 2005, 21:3674-3676.

30. Goff SA, Vaughn M, McKay S, Lyons E, Stapleton AE, Gessler D, Matasci N, Wang L, Hanlon M, Lenards A, Muir A, Merchant N, Lowry S, Mock S, Helmke M, Kubach A, Narro M, Hopkins N, Micklos D, Hilgert U, Gonzales M, Jordan C, Skidmore E, Dooley R, Cazes J, McLay R, Lu Z, Pasternak S, Koesterke L, Piel WH, et al: The iPlant Collaborative: Cyberinfrastructure for Plant Biology. Plant Sci: Front; 2011:2.

31. Blankenberg D, Kuster GV, Coraor N, Ananda G, Lazarus R, Mangan M, Nekrutenko A, Taylor J: Galaxy: a web-based genome analysis tool for experimentalists. In Curr Protoc Mol Biol. Edited by Ausubel FM, Brent R, Kingston RE, Moore DD, Seidman JG, Smith JA, Struhl K. Hoboken, NJ, USA John Wiley \& Sons, Inc; 2010

32. Giardine B, Riemer $C$, Hardison RC, Burhans R, Elnitski L, Shah $P$, Zhang $Y$ Blankenberg D, Albert I, Taylor J, Miller W, Kent WJ, Nekrutenko A: Galaxy: a platform for interactive large-scale genome analysis. Genome Res 2005 15:1451-1455

33. Goecks J, Nekrutenko A, Taylor J, Galaxy Team T: Galaxy: a comprehensive approach for supporting accessible, reproducible, and transparent computational research in the life sciences. Genome Biol 2010, 11:R86.

34. Ewen-Campen B, Shaner N, Panfilio KA, Suzuki Y, Roth S, Extavour CG: The maternal and early embryonic transcriptome of the milkweed bug Oncopeltus fasciatus. BMC Genomics 2011, 12:61.

35. Sloan DB, Keller SR, Berardi AE, Sanderson BJ, Karpovich JF, Taylor DR: De novo transcriptome assembly and polymorphism detection in the 
flowering plant Silene vulgaris (Caryophyllaceae). Mol Ecol Resour 2012, 12:333-343.

36. Hampton M, Melvin RG, Kendall AH, Kirkpatrick BR, Peterson N, Andrews MT: Deep Sequencing the Transcriptome Reveals Seasonal Adaptive Mechanisms in a Hibernating Mammal. PLOS ONE 2011, 6:e27021.

37. Babraham Bioinformatics: FastQC A Quality Control tool for High Throughput Sequence Data. [http://www.bioinformatics.babraham.ac.uk/ projects/fastac/]

38. FASTX-Toolkit. [http://hannonlab.cshl.edu/fastx_toolkit/]

39. Cahais V, Gayral P, Tsagkogeorga G, Melo-Ferreira J, Ballenghien M, Weinert L, Chiari Y, Belkhir K, Ranwez V, Galtier N: Reference-free transcriptome assembly in non-model animals from next-generation sequencing data: $D E$ NOVO NGS-BASED TRANSCRIPTOME ASSEMBLY. Mol Ecol Resour 2012, 12:834-845.

40. Kumar S, Blaxter ML: Comparing de novo assemblers for 454 transcriptome data. BMC Genomics 2010, 11:571.

41. Martin J, Bruno VM, Fang Z, Meng X, Blow M, Zhang T, Sherlock G, Snyder M, Wang Z: Rnnotator: an automated de novo transcriptome assembly pipeline from stranded RNA-Seq reads. BMC Genomics 2010, 11:663.

42. Hornett EA, Wheat CW: Quantitative RNA-Seq analysis in non-model species: assessing transcriptome assemblies as a scaffold and the utility of evolutionary divergent genomic reference species. BMC Genomics 2012, 13:361.

43. Mundry M, Bornberg-Bauer E, Sammeth M, Feulner PGD: Evaluating Characteristics of De Novo Assembly Software on 454 Transcriptome Data: A Simulation Approach. PLOS ONE 2012, 7:e31410.

44. Vijay N, Poelstra JW, Künstner A, Wolf JBW: Challenges and strategies in transcriptome assembly and differential gene expression quantification A comprehensive in silico assessment of RNA-seq experiments. Mol Ecol 2013, 22:620-634.

45. O'Neil ST, Emrich SJ: Assessing De Novo transcriptome assembly metrics for consistency and utility. BMC Genomics 2013, 14:465.

46. ABySS 1.3.5: Canada's Michael Smith Genome Sciences Centre. [http://www. bcgsc.ca/platform/bioinfo/software/abyss/releases/1.3.5]

47. Henschel R, Lieber M, Wu L-S, Nista PM, Haas BJ, LeDuc RD: Trinity RNA-Seq assembler performance optimization. In Proc 1st Conf Extreme Sci Eng Discov Environ Bridg EXtreme Campus Beyond. Chicago, Illinois: ACM; 2012:1-8.

48. Grabherr MG, Haas BJ, Yassour M, Levin JZ, Thompson DA, Amit I, Adiconis X, Fan L, Raychowdhury R, Zeng Q, Chen Z, Mauceli E, Hacohen N, Gnirke A, Rhind N, di Palma F, Birren BW, Nusbaum C, Lindblad-Toh K, Friedman N, Regev A: Full-length transcriptome assembly from RNA-Seq data without a reference genome. Nat Biotechnol 2011, 29:644-652.

49. McQuilton P, St Pierre SE, Thurmond J: FlyBase Consortium: FlyBase 101-the basics of navigating FlyBase. Nucleic Acids Res 2012, 40(Database issue):D706-D714.

50. Bactrocera dorsalis (ID 167923) - BioProject - NCBI. [http://www.ncbi.nlm.nih. gov/sra/?term $=366392]$

51. Punta M, Coggill PC, Eberhardt RY, Mistry J, Tate J, Boursnell C, Pang N Forslund K, Ceric G, Clements J, Heger A, Holm L, Sonnhammer ELL, Eddy SR, Bateman A, Finn RD: The Pfam protein families database. Nucleic Acids Res 2011, 40:D290-D301.

52. Wang X-W, Luan J-B, Li J-M, Bao Y-Y, Zhang C-X, Liu S-S: De novo characterization of a whitefly transcriptome and analysis of its gene expression during development. BMC Genomics 2010, 11:400.

53. Schwartz TS, Tae H, Yang Y, Mockaitis K, Van Hemert JL, Proulx SR, Choi J-H, Bronikowski AM: A garter snake transcriptome: pyrosequencing, de novo assembly, and sex-specific differences. BMC Genomics 2010, 11:694.

54. Bao B, Xu W-H: Identification of gene expression changes associated with the initiation of diapause in the brain of the cotton bollworm, Helicoverpa armigera. BMC Genomics 2011, 12:224.

55. Tweedie S, Ashburner M, Falls K, Leyland P, McQuilton P, Marygold S, Millburn G, Osumi-Sutherland D, Schroeder A, Seal R, Zhang H: The FlyBase Consortium: FlyBase: enhancing Drosophila Gene Ontology annotations. Nucleic Acids Res 2009, 37:D555-D559 (Database).

56. Jourdren L, Bernard M, Dillies M-A, Le Crom S: Eoulsan: a cloud computing-based framework facilitating high throughput sequencing analyses. Bioinforma Oxf Engl 2012, 28:1542-1543.

57. Zhao Q-Y, Wang Y, Kong Y-M, Luo D, Li X, Hao P: Optimizing de novo transcriptome assembly from short-read RNA-Seq data: a comparative study. BMC Bioinformatics 2011, 12(Suppl 14):S2.
58. Ang $Y$, Puniamoorthy $N$, Meier $R$ : Secondarily reduced foreleg armature in Perochaeta dikowi sp.n. (Diptera: Cyclorrhapha: Sepsidae) due to a novel mounting technique. Syst Entomol 2008, 33:552-559.

59. Concha C, Li F, Scott MJ: Conservation and sex-specific splicing of the doublesex gene in the economically important pest species Lucilia cuprina. J Genet 2010, 89:279-285.

60. DeWoody JA, Abts KC, Fahey AL, Ji Y, Kimble SJA, Marra NJ, Wijayawardena BK, Willoughby JR: Of contigs and quagmires: next-generation sequencing pitfalls associated with transcriptomic studies. Mol Ecol Resour 2013, 13:551-558.

doi:10.1186/1471-2164-15-188

Cite this article as: Melicher et al: A pipeline for the de novo assembly of the Themira biloba (Sepsidae: Diptera) transcriptome using a multiple k-mer length approach. BMC Genomics 2014 15:188.

\section{Submit your next manuscript to BioMed Central and take full advantage of:}

- Convenient online submission

- Thorough peer review

- No space constraints or color figure charges

- Immediate publication on acceptance

- Inclusion in PubMed, CAS, Scopus and Google Scholar

- Research which is freely available for redistribution

Submit your manuscript at www.biomedcentral.com/submit
() Biomed Central 